\title{
Editor-In-Chief
}

Fazlul Huq (Australia)

\section{Editorial Manager}

Md Shamsuddin Sultan Khan (Australia)

\section{Editorial Board Members}

Dr Oon Chern Ein (Malaysia)

Dr Aman Shah Bin Abdul Majid(Malaysia)

Dr Mohammed Khadeer Ahamed Basheer (Malaysia) Dr

Rosanani SM Anwarul Haque (Malaysia)

Dr Mohammed Adnan Iqbal (Pakistan)

Dr Loiy Elsir Ahmed Hassan (Malaysia)

Dr Chris Cazzonelli (Australia)

Prof Christoper Paris (Australia)

Dr Asaduzzaman Shishir (Bangladesh)

Dr Ali. Fareed. Jameel (Malaysia)

Dr. Muhammad Asif (Pakistan)

Dr. Elham Farsi (Iran)

Dr. Saad Albeaz Dham (Malaysia)

Dr. Armaghan Shafaei (Iran)

Dr. Malek Zihlif (Jordan)

Dr. Mouayed A. Hussein (Iraq)

Dr. Zeyad Nassar (Australia)

Dr. Fouad Saleih R. Al-Suede (Malaysia)

\section{Editorial Assiatant}

Nicholas Iannantuono (Canada) 


\section{Table of Contents}

Immunomodulatory and Antiangiogenic Mechanisms of Polymolecular Botanical Drug Extract C5OSEW5050ESA OS Derived from Orthosiphon stamineus,

Fouad Saleih R. Al-Suede1*, Mohamed B. Khadeer Ahamed1, Aman S. Abdul Majid2 Sultan Ayesh Mohammed Saghir3, Chern E. Oon4, Amin Malik Shah Abdul Majid1,5*

Published online 13 March 2021

Cannabis Sativa: A Remedy for Convulsion by Inhibition of GABAA Receptor and Significantly Delayed Onset of Seizure Latency and Death - An Experimental Validation and Molecular Docking.

Ben Enoluomen Ehigiator1, SamuelKelechi Mobisson2, Iheanyichukwu Wopara3*, Chinonye Cynthia Chibuife1, Uwaezuoke Chukwuekele Awarajih3, Harrison Ogheneochuko Eruotor3, pp. 207-217

Published online 30 October 2021

Immunomodulatory Effect of Tinospora cordifolia with Special Reference to Suppression of Cytokine Storm Induced in SARS-CoV-2

Aishath Thahuseen Waheed1, Thurga Ayavoo2,3, Karthikeyan Murugesan2,3, Fouad Saleih R. AL-Suede4,5, Ashok Gnanasekaran2,3*

pp. 218-225

Published online 10 November 2021

Phytochemicals with direct and/or indirect anti-angiogenic properties against various cancer types focusing on their mechanism of action

Ibrahim Al-deeb1,2,3, Julia Joseph1, Amin Malik Shah Abdul Majid2, Nozlena Abdul Samad1

pp. 226-233

Published online 11 November 2021 